\title{
Medizinische Versorgung in einer Aufnahmeeinrichtung für Asylbewerber
}

Die Häufigkeit von Rettungsdiensteinsätzen

Die Aufgabe, Flüchtlinge in Deutschland zu versorgen, hat in der zweiten Jahreshälfte 2015 eine unerwartete Größenordnung erreicht. Eine Herausforderung für den Rettungsdienst besteht darin, neben der Abwehr von Lebensgefahr oder schwerer gesundheitlicher Schäden, bei vermeintlichen Notfällen Flüchtlinge in eine angemessene Weiterversorgung zu lenken.

\section{Die medizinische Situation}

Flüchtlinge und Migranten weltweit und in Deutschland Weltweit waren im Jahr 2014 fast 60 Mio Menschen auf der Flucht. Davon hatten 19,5 Mio eine Staatengrenze überschritten und galten deshalb nach völkerrechtlicher Definition als Flüchtlinge. Da die meisten Flüchtlinge lediglich in ein angrenzendes Nachbarland fliehen, leben 9 von 10 Flüchtlingen (86\%) in sogenannten „Entwicklungsländern“. Kriegerische Konflikte und andere Ursachen für Flucht und Vertreibung finden häufiger in unmittelbarer Nähe Europas statt und die Zahl von Menschen, die nach Europa flüchten, steigt. In Deutschland wurden 2013127000 Asylanträge gestellt, in 2014203000 und in 2015 bis Ende November 425000. [1, 2, 3] Dieser Anstieg spiegelt die Zahl der in Deutschland befindlichen Flüchtlinge nur unvollständig wieder, denn die Wartezeiten bis zum Stellen eines Asylantrages haben zugenommen. Verfolgte und Vertriebene werden häufiger Patienten des Rettungsdienstes in Deutschland. Deren besondere Situation und die Beschränkungen bei der allgemeinen Gesundheitsversorgung sind auch für den Rettungsdienst relevant.

Krankheitsspektrum ist ähnlich Menschen mit Migrationshintergrund haben insgesamt ein ähnliches Krankheitsspektrum wie die Mehrheitsbevölkerung, auch wenn Krankheiten mit unterschiedlicher Häufigkeit auftreten. Dieses spiegelt sich auch bei der primärärztlichen Versorgung in Deutschland ankommender Flüchtlinge wieder [4]. Sorgen von Notärzten und Rettungsfachpersonal vor Infektionsgefährdung bei der Versorgung von Flüchtlingen sind deshalb nicht mehr und nicht weniger begründet, wie bei der Versorgung anderer Patienten. Die Auswahl der Hygienemaßnahmen sollte sich nicht am Aufenthaltsstatus des Patienten orientieren, sondern an der Einsatzsituation, der Vorgeschichte und den medizinischen Befunden.

Spezifische Gesundheitsrisiken bei Migranten und besonders bei Flüchtlingen Die unterschiedliche Häufigkeit des Auftretens verschiedener Krankheiten bei Menschen mit Migrationshintergrund resultiert aus spezifischen Gesundheitsrisiken, die bei Deutschen ohne Migrationshintergrund nur in Ausnahmefallen vorkommen oder selten geworden sind [5]. Von derartigen Gesundheitsproblemen sind besonders Flüchtlinge betroffen. Mangelnde oder fehlende Gesundheitsversorgung im Herkunftsland und während der Reise, Folter, lebensgefährliche Reisebedingungen und weitere Einschränkungen bei den Grundbedürfnissen verursachen besonders bei Flüchtlingen spezifische körperliche und psychische Gesundheitsprobleme [6, 7].

Anderes Nutzungsverhalten bei Gesundheitsleistungen Menschen mit Migrationshintergrund weisen auch bei Gesundheitsproblemen, die denen der Mehrheitsbevölkerung ähneln, ein anderes Nutzungsverhalten bei Inanspruchnahme von Gesundheitsleistungen auf. Dieses unterschiedliche Nutzungsverhalten ist ein Gegenstand der Migrationsforschung und kann teilweise durch kulturelle Unterschiede erklärt werden [5, 8]. Bei Flüchtlingen ist ein weiterer Effekt relevant. Unterschiedliche strukturelle Rahmenbedingungen regeln den Zugang zur Gesundheitsversorgung. Im Vergleich zur Mehrheitsbevölkerung ist dieser eingeschränkt $[5,7,9]$

\section{Die Situation in einer \\ Aufnahmeeinrichtung}

Beispiel Braunschweig In der Stadt Braunschweig befindet sich ein Standort der Niedersächsischen Landesaufnahmebehörde (LAB), der für 750 Bewohner konzipiert ist. Hier werden Flüchtlinge und Asylbewerber aufgenommen und erfasst. Sie sollen nach max. 3 Monaten zur längerfristigen Unterbringung auf niedersächsische Landkreise und Städte verteilt werden. Deshalb ist die Aufenthaltsdauer in der LAB grundsätzlich kurz. Während des Aufenthaltes in der LAB erfolgt auch die vorgeschriebene Gesundheitsuntersuchung auf übertragbare Krankheiten durch das Gesundheitsamt. Eine Gesundheitsstation bietet ein niedrigschwelliges Versorgungsangebot. Sie wird von medizinisch geschultem Personal besetzt. Bis Ende September 2014 war diese Gesundheitsstation $40 \mathrm{~h}$ pro Woche erreichbar. Seit Oktober 2014 wird sie kontinuierlich betrieben. Hausärztliche Konsultationen werden durch die Gesundheitsstation gebahnt. Sie erfolgen an 2 Nachmittagen der Woche in der Gesundheitsstation oder während der Praxiszeiten in einer Hausarztpraxis in der Nähe. In Einzelfällen werden Facharzttermine organisiert. Außerhalb der Sprechzeiten steht der ärztliche Bereitschaftsdienst der kassenärztlichen Vereinigung zur Verfügung. Der Sozialdienst, mit dem jeder Bewohner im Rahmen des Aufnahmeprozesses Kontakt hat, führt bei Verdacht auf eine posttraumatische Belastungsstörung (PTBS) ein strukturiertes Screening durch [10]. Seit Oktober 2014 werden die Betroffenen bei einer wahrscheinlich vorliegenden PTBS für 3 Untersuchungstermine an die Psychotherapieambulanz der Tech- 


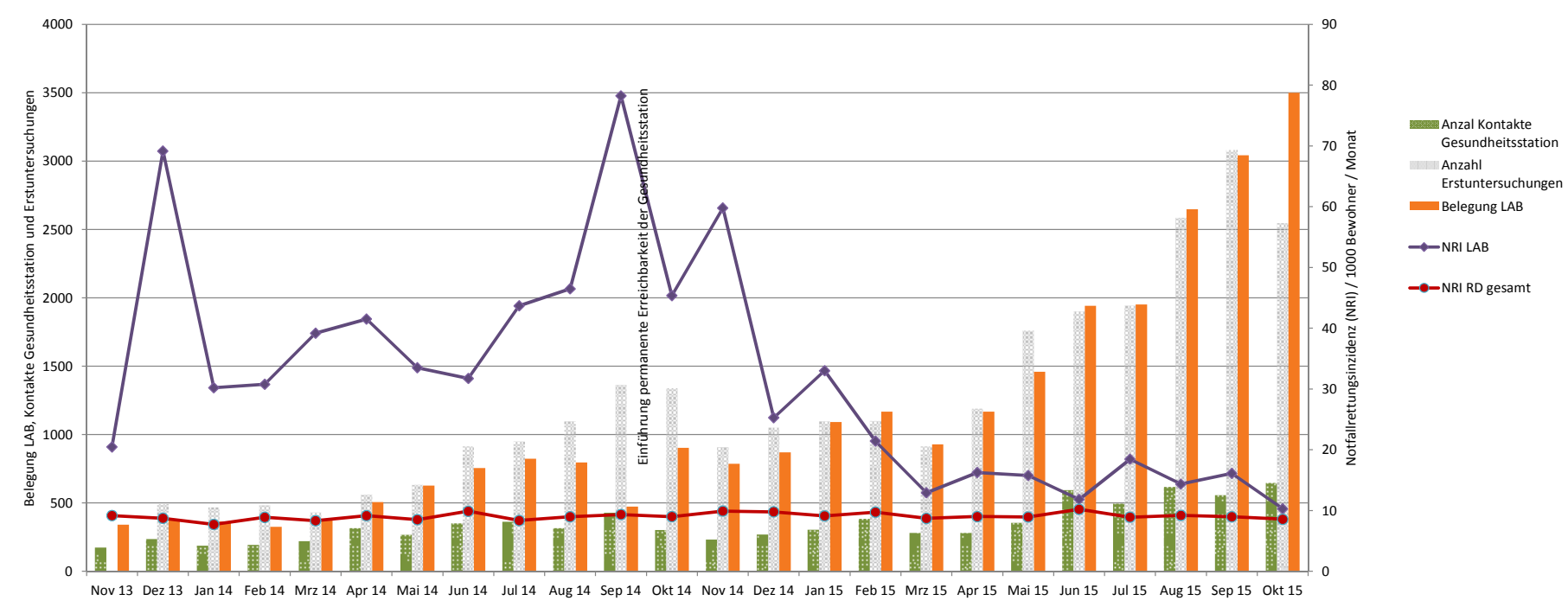

Abb. 1 Monatlicher Verlauf der Notfallrettungsinzidenz (NRI) und weiterer Versorgungszahlen.

nischen Universität Braunschweig überwiesen. Bei potenziell lebensbedrohlichen Situationen wird Notfallrettung durch die Gesundheitsstation, durch andere Mitarbeiter der LAB oder durch die Bewohner selbst über Notruf eingeleitet. Der Rettungsdienst der Stadt Braunschweig stellt Notfallrettung und qualifizierten Krankentransport im Stadtgebiet sicher. Die Stadt Braunschweig umfasst etwa $192 \mathrm{~km}^{2}$ mit etwa 250000 Einwohnern. Jährlich werden über 60000 Einsätze geleistet, davon sind mehr als die Hälfte qualifizierte Krankentransporte.

\section{Notfallrettung und medizini- sche Versorgung}

Die Häufigkeit von Rettungsdiensteinsätzen in der LAB wird regelmäßig ermittelt Um eine mögliche Abhängigkeit von der weiteren Versorgung $\mathrm{zu}$ betrachten werden neben Daten der Rettungsleitstelle auch Belegungszahlen der LAB, Fallzahlen der Gesundheitsstation in der LAB und die Anzahl von Erstuntersuchungen des Gesundheitsamtes retrospektiv ausgewertet. Im Zweijahreszeitraum vom 1.12.2013 bis zum 30.11.2015 gab es in der LAB durchschnittlich 29,8 Rettungsdiensteinsätze pro Monat. Davon waren 26,6 (89,2\%) Notfallrettungseinsätze und 3,2 (10,8\%) Krankentransporte. Durchschnittlich 6,4 Einsätze erfolgten mit notärztlicher Beteiligung (24,1\% der Notfallrettungseinsätze). Im gesamten Rettungsdienstbereich erfolgten in diesem
Zweijahreszeitraum monatlich etwa 5100 Rettungsdiensteinsätze. 2250 waren Notfallrettungseinsätze, davon erfolgten 575 (25,6\%) mit Notarzt. Krankentransporte waren in der LAB also selten, die Notarztquote in der LAB und im gesamten Rettungsdienstbereich war vergleichbar. Weitere Vergleiche und Diskussionen beschränken sich deshalb auf Notfallrettungseinsätze. In der Gesundheitsstation erfolgten im Zweijahreszeitraum im Durchschnitt 349 Patientenkontakte pro Monat. Davon waren 235 Erstkontakte und 114 Folgekontakte. Daten über Untersuchungen durch das Gesundheitsamt waren ab 2014 verfügbar. Im Zeitraum vom 1.1.2014 bis zum 30.6.2015 wurden durchschnittlich 1252 Erstuntersuchungen pro Monat durchgeführt.

Inzidenz Für die Berechnung der Inzidenz von Notfallrettungseinsätzen in der LAB wurde deren durchschnittliche Belegungszahl verwendet. Die Ermittlung dieser Belegungszahl erfolgt auf Basis einer Aufenthaltsdauer ab dem ersten Werktag nach Ankunft bis zur Verlegung. Menschen, die an diesem Erfassungstag einer anderen Behörde zugeordnet werden oder verlegt werden, bleiben bei der Berechnung der durchschnittlichen Belegung unberücksichtigt. Für die Inzidenz im gesamten Rettungsdienstbereich wurde die Einwohnerzahl zugrunde gelegt. Die durchschnittliche Belegungszahl der LAB im Untersuchungszeitraum betrug 1104 Bewohner, die Not- fallrettungsinzidenz (NRI) in der LAB 24,1 Einsätze/Bewohner/Monat, im gesamten Rettungsdienstbereich 9,0 Einsätze/Bewohner/Monat.

Die monatliche Entwicklung der NRI, der Zahl der Erstuntersuchungen, der Patientenkontakte in der Gesundheitsstation und der Belegungszahl der LAB sind in der Grafik dargestellt (Abb. 1). Daran ist erkennbar, dass die NRI in der LAB zunächst deutlich höher als im gesamten Rettungsdienstbereich war. Die NRI sank nach Einführung einer kontinuierlichen Erreichbarkeit der Gesundheitsstation auf ein Niveau in der Größenordnung des gesamten Rettungsdienstbereiches und blieb bei Überbelegung unverändert.

Ist die Einsatzhäufigkeit in Braunschweig repräsentativ? Die NRI lag in Braunschweig mit 108/1000 Einwohner/Jahr deutlich höher als der bundesweite Durchschnitt von 70/1000 Einwohner/Jahr. Die NA-Inzidenz lag mit 28/1000Einwohner/Jahr Notfallrettungsinzidenz unter dem bundesweiten Mittel von 35/1000Einwohner/Jahr. Ursachen für diese Unterschiede sind anhand der vorliegenden Daten nicht zu ermitteln. Neben soziodemografischen Faktoren können auch andere Alarmierungsschwellen für Notfallrettung und Notarzteinsatz zu diesen Unterschieden beigetragen haben [11, 12]. Die Häufigkeit von Notarzteinsätzen hängt von soziodemografischen Faktoren der Bevölkerung ab und wird durch den Zugang 
zu ambulanter Versorgung beeinflusst [11]. Dieses gilt auch für vermeidbare stationäre Behandlungen [13, 14]. Ähnliches könnte auch für die Gesamtheit der Notfallrettungseinsätze vermutet werden. Dabei kann die NRI nicht als Indikator für die medizinisch-soziodemografische Situation oder den Zugang zu ambulanter Versorgung angesehen werden, sondern allenfalls als ein unspezifischer zusätzlicher Eindruck von einer komplexen Gesamtsituation.

Patientenkontakte in der LAB und in der Mehrheitsbevölkerung Die Inzidenz von Patientenkontakten in der Gesundheitsstation der LAB war mit 5/Bewohner/Jahr im Vergleich zu Arztkontakten der Gesamtbevölkerung niedrig. Im Jahr 2007 betrug die bundesweite Anzahl an Arztkontakten im Durchschnitt 17 pro Versicherten, bei jungen Männern sieben pro Jahr [15]. Die niedrige Häufigkeit von Patientenkontakten in der LAB kann kaum mit einem geringeren Versorgungsbedarf der versorgten Population erklärt werden. Vielmehr würden mangelnde oder fehlende Gesundheitsversorgung im Herkunftsland und während der Reise, Folter, lebensgefährliche Reisebedingungen und weitere Einschränkungen bei den Grundbedürfnissen spezifische körperliche und psychische Gesundheitsprobleme und damit eher einen höheren Versorgungsbedarf erwarten lassen [5-7]. Diese Diskrepanz könnte dadurch erklärbar sein, dass Gesundheitsprobleme mit niedriger Dringlichkeit wegen der kurzen Aufenthaltsdauer in der LAB kaum präsentiert werden. Das Asylbewerberleistungsgesetz, welches den Zugang zur Gesundheitsversorgung im Wesentlichen auf Akutsituationen einschränken soll, kann die niedrige Inzidenz von Patientenkontakten nicht erklären, denn auch abgewiesene Bewohner würden als Kontakte erfasst werden [7, 9]. Neben der kurzen Aufenthaltsdauer bleibt u.a. der Umstand, dass Krankschreibung als häufiger Anlass für einen Arztbesuch von jungen Erwachsenen in der Gesamtbevölkerung bei Bewohnern der LAB entfällt. Vor diesem Hintergrund scheint die niedrige Häufigkeit von Patientenkontakten plausibel. Die höhere NRI deutet darauf hin, dass der Zugang zur notfallmedizinischen Versorgung für Be- wohner der LAB nicht schlechter als in der Gesamtbevölkerung zu sein scheint. Soweit wären die diskutierten Fallzahlen mit einer Sicherstellung der Versorgung dringender Probleme vereinbar. Allerdings dürften die in der LAB präsentierten Gesundheitsprobleme nicht repräsentativ für Flüchtlinge und Asylsuchende in Deutschland sein.

Eine niedrigschwellige medizinische Versorgung als Lotse im Gesundheitswesen Die Reduzierung der NRI nach Einführung einer kontinuierlichen Erreichbarkeit der Gesundheitsstation deutet darauf hin, dass diese nach einer mehrmonatigen Etablierungsphase in der Lage sein kann, die medizinische Versorgung der LAB Bewohner so zu lenken, dass Notfallrettungseinsätze reduziert werden können und dass dieses auch bei einer massiven Überbelegung möglich ist. Ein ähnlicher Effekt wurde für Notarzteinsätze und die Inanspruchnahme des ärztlichen Bereitschaftsdienstes nach Etablierung täglicher Sprechstunden in einer Würzburger Flüchtlingsunterkunft gezeigt [16]. Einen kausalen Zusammenhang beweist unsere retrospektive Auswertung nicht. Ebenso wenig kann eine Aussage getroffen werden, ob eine derartige Reduzierung der Häufigkeit von Notfallrettungseinsätzen eine Verbesserung oder eine Verschlechterung der Versorgungsqualität bewirkt hat. Eine Reduzierung von unnötigen Transporten und von Überdiagnostik würde die Versorgungsqualität verbessern, eine Verzögerung notwendiger notfallmedizinischer Versorgung hätte negative Auswirkungen. Hinweise auf derartige Effekte sehen wir in unseren Ergebnissen nicht. Da die Notfallrettungsinzidenz weiterhin über der des gesamten Rettungsdienstbereiches liegt, können wir keinen Hinweis auf einen eingeschränkten Zugang zur notfallmedizinischen Versorgung für die Bewohner der LAB erkennen. Wir vermuten, dass die permanente Verfügbarkeit der Gesundheitsstation zu einem insgesamt effizienteren Ressourceneinsatz geführt hat und halten analog zum Würzburger Modell positive Einflüsse auf die Versorgungsqualität für möglich, die ohne Verursachung von Mehrkosten erreichbar sein könnte [16, 17].

\section{Fazit}

Eine Aufgabe bei der Versorgung von Asylsuchenden besteht für Notärzte darin, spezifische gesundheitliche Probleme, die bei der Mehrheitsbevölkerung nur in Ausnahmefallen vorkommen oder selten geworden sind, zu erkennen, zu bewerten und einer angemessenen Versorgung zuzuführen. Dazu ist die Kenntnis der lokalen Versorgungsstrukturen hilfreich. Über die gesundheitliche Lage von Asylsuchenden in Deutschland gibt es kaum Informationen [18]. Weitergehende Studien zum Zugang zu medizinischer Versorgung sollten auch den Rettungsdienst betrachten.

\section{Autoren}

A. Günther ${ }^{1,2}$, B. Piest ${ }^{3}$, I. Herrmann ${ }^{4}$, R. Schulte ${ }^{5}$, B. Buhr-Riehm ${ }^{6}$, K. Siems ${ }^{5}$, K. Konstantin ${ }^{1,2,7}$

\section{Institute}

${ }^{1}$ Berufsfeuerwehr der Stadt Braunschweig, Rettungsdienst

${ }^{2}$ Klinik für Anästhesiologie, Klinikum Braunschweig gGmbH

${ }^{3}$ Hausarztpraxis Bernhard Piest und Andreas Luther, Braunschweig

${ }^{4}$ Gesundheitsstation, Die Johanniter, Landesaufnahmebehörde Niedersachsen, Standort Braunschweig

${ }^{5}$ Landesaufnahmebehörde Niedersachsen

${ }^{6}$ Gesundheitsamt der Stadt Braunschweig

${ }^{7}$ Médecins Sans Frontières (MSF) - Ärzte ohne Grenzen, Deutsche Sektion e.V., Vorstand

\section{Korrespondenzadresse}

Dr. Andreas Günther

Ärztlicher Leiter Rettungsdienst der Stadt Braunschweig, Berufsfeuerwehr, Stadt Braunschweig

E-Mail: andreas.guenther@braunschweig.de

Beitrag online zu finden unter http://dx.doi. org/10.1055/s-0042-100238 